\title{
Full Length Spine CT and MRI
}

\section{Tae-Keun Ahn'1, Stephane Bourret'2, Wendy Thompson², Cecile Roscop², Thibault Cloché2, Jean-Charles Le Huec ${ }^{2}$}

${ }^{1}$ Department of Orthopedic Surgery, CHA Bundang Medical Center, CHA University, Seongnam, Republic of Korea

${ }^{2}$ Vertebra Spine Unit, Polyclinique Bordeaux Nord Aquitaine, Bordeaux, France

Email: jclehuec1@gmail.com

How to cite this paper: Ahn, T.-K., Bourret, S., Thompson, W., Roscop, C., Cloché, T. and Le Huec, J.-C. (2020) Full Length Spine CT and MRI. International Journal of Clinical Medicine, 11, 270-281.

https://doi.org/10.4236/ijcm.2020.115028

Received: April 16, 2020

Accepted: May 12, 2020

Published: May 15, 2020

Copyright (C) 2020 by author(s) and Scientific Research Publishing Inc. This work is licensed under the Creative Commons Attribution International License (CC BY 4.0).

http://creativecommons.org/licenses/by/4.0/

\begin{abstract}
Spine is a complex and long structure in the human body. Visualization of the spine is essential to treat and manage spine disease and commonly requires further imaging modalities such as computed tomography (CT) and magnetic resonance imaging (MRI). In most clinical fields, spine CT and MRI examinations are focused on the region of interest. However, spine is composed of cervical, thoracic, lumbar, sacrum and coccyx and sometimes demands examination of entire structure as well as regional spine depending on disease, patient's state and physician's decision. This review considers the available literature to describe when and how full length spine evaluation by CT and MRI is applied according to each spinal disease such as spinal trauma, deformity, infection, axial spondyloarthropathy and metastatic tumor.
\end{abstract}

\section{Keywords}

Full Length Spine CT, Full Length Spine MRI, Trauma, Deformity, Infection, Axial Spondyloarthropathy, Metastasis

\section{Introduction}

There are multiple imaging modalities to evaluate the spine. The type of imaging tool for the spine depends on the type of disease, the amount of radiation hazard, contraindications and any allergy to contrast.

Among the multiple imaging strategies, computed tomography (CT) is a reliable method to evaluate the spine and has better sensitivity for bony abnormality than radiography or magnetic resonance imaging (MRI) [1]. In the past, the time factor limited the use of CT. However, owing to the development of fast spiral CT and multidirectional CT (MDCT), the whole spine can be examined in very short time [2]. CT is less sensitive to patient's movement than MRI [3]. Now, it is widely used to assess trauma, deformity, metastasis, pre and post-operative 
patients.

MRI is one of the most sensitive and specific modalities to visualize the spine. Furthermore, there are no risks of radiation hazard. It provides information about the spinal neural structures including cord, cauda equina and roots, the vertebral bodies and intervertebral disc. Multiple sequences can be obtained in different planes and each sequence helps in evaluating various changes of the spine. The most commonly using sequences include sagittal and axial T1 and T2, and a sagittal STIR (short tau inversion recovery). Post-contrast T1 weighted images are useful for evaluating spinal infections, postoperative complications, vascular malformation, primary spinal tumors, and metastases. STIR sequences are beneficial in assessing marrow edema, which demonstrates an increased signal on these sequences [4].

In most cases, spine CT and MR images are taken focusing on the region of interest where the pathology exists. However, full length spine examination is more helpful in detailed evaluation and early detection of disease and trauma being able to involve the entire spine (Figure 1). Therefore, the aim of this review was to describe when and how full length spine evaluation through CT and MRI is applied.

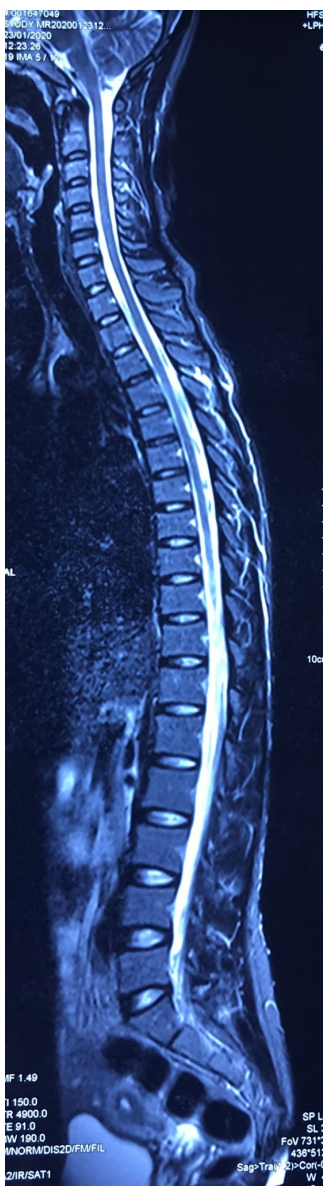

Figure 1. Normal sagittal T2 weighted magnetic resonance image of full length spine. 


\section{Methodology}

A literature review using PubMed and Google Scholar was performed. To identify relevant studies, search was conducted on studies in English published between 2000 and February, 2020 using various combinations of the following key words: "full length spine", "entire spine", "whole spine", "imaging", "computed tomography". "magnetic resonance imaging". Based on this search, 127 studies were initially detected. After excluding articles about rare disease, technical aspect of CT and MRI, measurement and imaging protocol, 56 articles were selected. After an internal peer review with all the authors of this article and a supplementary search that involved bibliographic screening and citation tracking associated with individual spine pathology, 24 articles of interest were finally selected (Figure 2). Those articles are categorized as following sections: "Trauma", "Deformity", "Infection", "Axial spondyloarthropathy" and "Spinal metastasis".

\section{Contributions of Full Length Spine CT and MRI}

\subsection{Trauma}

When trauma to the spine occurs, according to the findings of physical examination and simple radiography, targeted CT and/or MRI is usually performed on the suspected spine as a further imaging work up. However, if the patient sustains
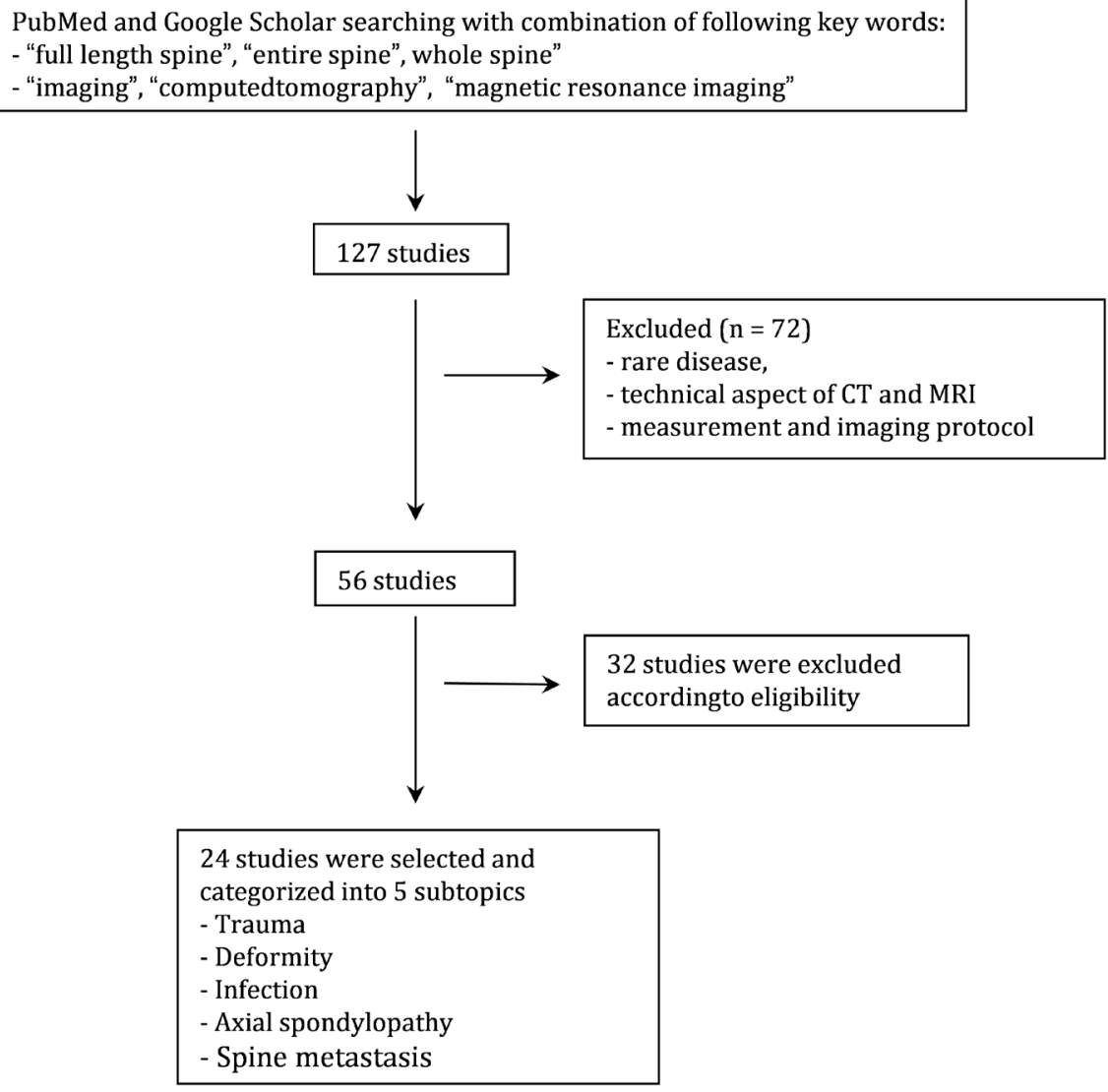

Figure 2. Methods of relavant study selection. 
high energy trauma or multiple trauma, those spine imaging can be insufficient. In high energy or multiple trauma patients, after the patient's condition is stabilized, unforeseen injuries to the spine should be delineated accurately because the symptoms of a spinal fracture may be masked by concomitant injuries, patient's state of consciousness (Figure 3), spinal cord injury or shock [5] [6]. In these situations, it requires full length spine evaluations with CT and/or MRI rather than simple radiographs, targeted CT or MRI.

In the patients with high energy blunt trauma, Deunk et al. reported that the non-targeted CT detected $8.2 \%$ more spine injuries unrevealed by simple radiographs than the targeted CT [7]. In a prospective study, spinal fractures were prevalent in $30.2 \%$ of cases of high-energy trauma. Among patients with a cervical fracture, $37.5 \%$ had a fracture that unrevealed on radiographs of the cervical spine but were first diagnosed by a CT scan. Patients with a thoracolumbar fracture did not have clinical symptoms of a fracture in $14 \%$, but had a fracture revealed by CT. Therefore, Takami et al. postulated that the full length spine CT should be taken to evaluate high energy trauma patients [6].

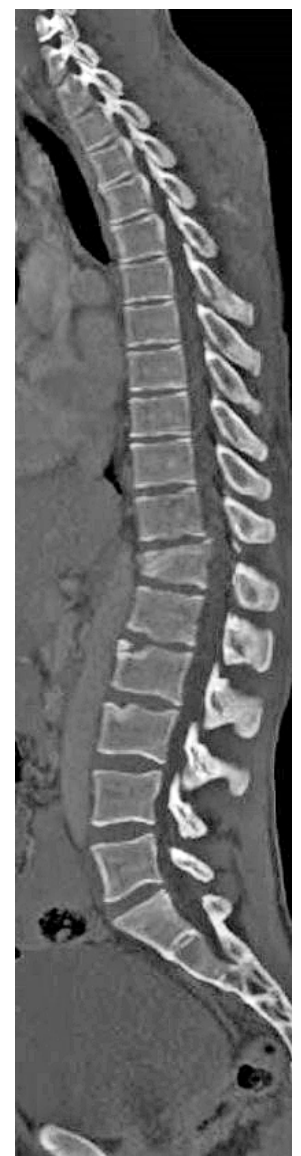

Figure 3. Sagittal full length spine computed tomography image showing Denis B type T12 fracture with widened interspinous space. A 29-year-old man sustained an injury from vehicle-pedestrian accident. The patient was unconscious in the emergency room. 
Using full length MRI in spine trauma patients remains controversial. Atsina et al. described that full length MRI is not usually recommended in patients with spine trauma. When the full length MRI is performed in patients with blunt trauma after full length spinal CT, most additional spinal injuries detected on MRI were bone contusions and mild compression fractures. These additional spinal diagnoses are unlikely to change the management or treatment [8]. Therefore, the targeted MRI is more useful in patients with high energy blunt trauma. However, Kanna et al. reported that full length sagittal T2 weighted images help diagnose multi-level non-contiguous spinal injuries in spinal trauma patients and can be performed in approximately 4 minutes without radiation hazard [9].

\subsection{Deformity}

Spinal deformity is a complex disease with different three-dimensional abnormalities that often involve the entire spine [10]. In cases with a complex or severe spinal deformity, full length spine CT should be taken of the entire area of spine including the involvement segment of the spine deformity because radiography alone is insufficient to visualize complicated abnormalities of spinal deformity [11]. Furthermore, in comparison with radiography, full length spine CT scanning provides additional information about extent of rotation of the spine, segmentation defects, three-dimensional reconstruction and detection of bony spur in diastomatomyelia and associated congenital anomalies of ribs, scapula, and pelvis [12].

Full length spine CT is also useful for surgical planning. Current surgical techniques for deformity correction is making surgeons capable of correcting the coronal, sagittal and rotational aspects by the $3 \mathrm{D}$ assessment of spinal deformity [13]. Multiplanar reconstruction of the axial CT images is important, especially when trying to understand the anatomy of deformity and allows visualizing levels where osteotomies or resections are planned. The CT scan allows surgeons to understand the pedicle anatomy including the width, depth, and trajectory of the pedicles. During a deformity surgery, caution must be placed when placing screws in the pedicles, especially on the concave side of curvature where pedicles are extremely narrow and the spinal cord is vulnerable to the iatrogenic injuries due to restricted epidural space [14]. Preoperative full length spine CT in this context has been recommended because of the high probability of the presence of narrow pedicles. It also allows surgeons to identify pseudarthroses if the patients underwent previous fusion surgery. Postoperative full length spine CT is considered for patients with a new neurologic deficit after surgery. Postoperative CT helps to find the malposition of pedicle screws that develops postoperative neurological symptoms [15].

Full length spine MRI usually is obtained in patients with spinal deformity who develop neurologic symptoms or are planned to be operated. MRI provides good visualization of neurological structures, and helps determine appropriate levels of surgery, and can assist in the evaluation of disc degeneration when fu- 
sion extension is considered [16]. MR imaging is also essential for the patients with an unusual curve pattern and for those patients who should be assessed on the neural axis. It is important to understand whether syrinx, tethering of the spinal cord, intramedullary tumors or Chiari malformations are present. The presence of those intraspinal anomalies without neurological findings in idiopathic scoliosis has been estimated between $4 \%$ and $26 \%$ and associated with neurological complications resulted from correction surgery [17]. Stenosis or cord impingement secondary to severe deformity can be visualized by full length MRI. In kyphoscoliosis, the level of cord compression should be identified preoperatively and always on the concave pedicle. Those compressions may require a resection of that pedicle with the remaining body during deformity surgery [11] [15].

\subsection{Infection}

The diagnosis of spinal infection can be difficult, and even delayed because clinical manifestations can be varied and equivocal. Clinical physicians have relied heavily on imaging modalities due to difficulty in detecting spinal infection. Among imaging modalities, MRI plays a pivotal role in the diagnosis and management of spinal infection with a sensitivity, specificity, and accuracy above $90 \%$ and the ability to evaluate the spine neural structures [18].

If disease process of spinal infection is strictly localized, it is not necessary that the entire spine is examined. However, identification of one infected site in the spine cannot rule out the infection in multiple region of the spine. The areas adjacent to anterolateral vertebral endplates has abundant vascular supply and are most common regions where pyogenic, tuberculous, and other spinal infections occur primarily. Associated edema is commonly pronounced and affects the entire vertebral body and intervertebral disc [19]. If there is epidural involvement associated with neurologic symptoms, it is recommended to take full length spine images under these circumstances because epidural abscesses can spread extensively [20]. Among spinal infections caused by various organisms, mycobacterium tuberculosis are notorious for multifocality, and subligamentous spread of infection to multiple levels is one of the imaging hallmarks of the disease. The infection typically starts in the anterior aspect of the vertebral body adjacent to the intervertebral disc and then spreads to the vertebral bodies under the anterior longitudinal ligaments involving multiple adjacent vertebrae. Through valveless vein system, it can also make non-contiguous lesion in another spine levels other than where the infection started [21].

The incidence of multifocal spinal infection has varied from around $4 \%$ to $23 \%$. Mann et al. evaluated 24 patients with spondylitis and found 21 patients had single level infections, while 1 (4\%) had multi-segmental infection [22]. Ledermann et al. found 7 (16\%) out of 44 of patients with disc infection had involvement at several spinal levels [23]. Cox et al. reported that 82 patients with single-level infection performed full length MRI examination and 19 (23\%) pa- 
tients had additional non-continuous sites of infection. Remote levels of spondylodiscitis were also present in 11 patients (13\%) [20]. In cases of multifocal spinal tuberculosis, the reported incidence is variable, ranging from $1.1 \%$ to $71.4 \%$. Kaila et al. reported a very high incidence of $71.4 \%$ [24], but their data is likely overestimated due to the small number of cases presented. According to a retrospective study, 47 (25.1\%) of 187 patients with spinal tuberculosis had multifocal spinal tuberculosis [25].

In the spinal infection, full length spine MRI can be used as a diagnostic screening test to detect multifocal lesions even if patients do not have clinical symptoms and provides early detection of spinal infection preventing progression of osteomyelitis. In regard to the management and treatment of spinal infection, the detection of an epidural abscess or other infective lesions remote from the site of known uncomplicated spinal infection will likely result in a change of management and treatment from conservative to surgical [26].

\subsection{Axial Spondyloarthropathy}

In the management and treatment of axial-spondyloarthropathy (SpA), the benefit of early detection before structural damage begins is of importance. However, clinical findings of early axial-SpA are not specific and assessment can be challenging as the structural components of the spine is not easy to assess due to the deep location. Furthermore, current imaging modalities such as radiographs, $\mathrm{CT}$, and bone scintigraphy have shown limitations for use in early detection of axial-SpA. Both CT and bone scintigraphy expose the patient to high levels of ionizing radiation; bone scintigraphy showed low specificity and CT cannot detect until structural bone changes have progressed [27]. On the other hand, MRI has shown high sensitivity and specificity of imaging tools to pre-structural early inflammatory changes taking the presentation of bone marrow edema adjacent to joints and discs, entheses, and ligaments [28].

Although MRI of the sacroiliac (SI) joints has traditionally been used in suspected axial-SpA, this may be inadequate. Inflammatory lesions in the spine have been found most commonly in the thoracic spine besides SI joints. Full length spine MRI highlighted the frequent involvement of the thoracic spine, with emphasis on the most lateral sagittal aspects of vertebral endplates and costovertebral junctions. About 23\% of ankylosing spondylitis patients with clinically active disease only have inflammatory spinal lesions and no evidence of active inflammatory sacroiliitis, even in very early disease. Cervical spine involvement is also common in ankylosing spondylitis. For these reasons, full length spine MRI of the whole spine including SI joints is an essential tool in the diagnosis, management and prognosis of axial-SpA [29].

Full length spine MRI has been integrated into recommendations for the staging and therapeutic response evaluation in ankylosing spondylitis. It has been used to evaluate the therapeutic response showing the transition from active enthesitis with bone marrow edema to quiescent fatty infiltration of the 
bone marrow [30].

\subsection{Spine Metastasis}

Plain radiography, CT, and MRI comprise the core imaging modalities for patients with vertebral metastases. CT provides cross sectional images, allows for entire spine imaging, is suitable to visualize cortical and trabecular bone, and is more sensitive than conventional radiography. CT scans can detect a bony metastatic lesion up to 6 months earlier than an X-ray. However, when compared to MRI, Buhmann et al. found the diagnostic accuracy of MRI (98.7\%) to be significantly superior to MDCT (88.8\%) for the detection of osseous metastases. Sensitivity was significantly lower for MDCT (66.2\%) than for MRI (98.5\%) [31]. Moreover, cortical destruction may be difficult to be detected in CT exam when osteoporosis or degenerative changes occur. There is also an inherent associated risk of radiation exposure. Changes in bone-marrow are fundamental to the sensitivity of MRI in the detection of sites of skeletal metastases in the spine. The combination of unenhanced T1-weighted spin echo and STIR sequences have shown to be most useful for the detection of bone marrow abnormalities and are able to discriminate benign from malignant bone marrow changes [32].

Therefore, contrast-enhanced MRI of the entire spinal axis is the current standard for the diagnosis and evaluation of spinal column metastases. If the MRI examination is limited to the spine region of interest, and bone marrow metastases outside the image volume can be missed. Full length spine MRI is often imperative to visualize multiple levels of spinal involvement with asymptomatic disease or the large amount of bone destruction on plain radiography. Full length spine MRI including pelvis has been used to detect bone metastases and hematologic malignancies. In prostate cancer, full length spine MRI including pelvis is highly sensitive for the detection of metastases in the axial skeleton (Figure 4). In multiple myeloma, the axial skeleton approach with entire spine and pelvis is recommended in the staging system [33].

After radiation therapy, MRI appears to be a powerful tool for differentiating post-therapeutic changes from tumor recurrence. CT is not used routinely during follow-up, but may be necessary for surveying osteoblastic, osteolytic or mixed lesions. MRI is also useful for evaluation of paravertebral masses and epidural extension [34].

\section{Summary}

Full length spine CT and MRI plays a significant role in diagnosis and management in spinal trauma and disease. Full length spine CT helps diagnose unforeseen injuries to the spine that may be missed due to concomitant or patient conditions in high energy and multiple trauma patients. In the patient with complex or severe deformity, full length spine CT facilitates to analyze deformed anatomy and establish preoperative plan. Neurological structures and intraspinal anomalies can be visualized by full length MRI, thereby can prevent 

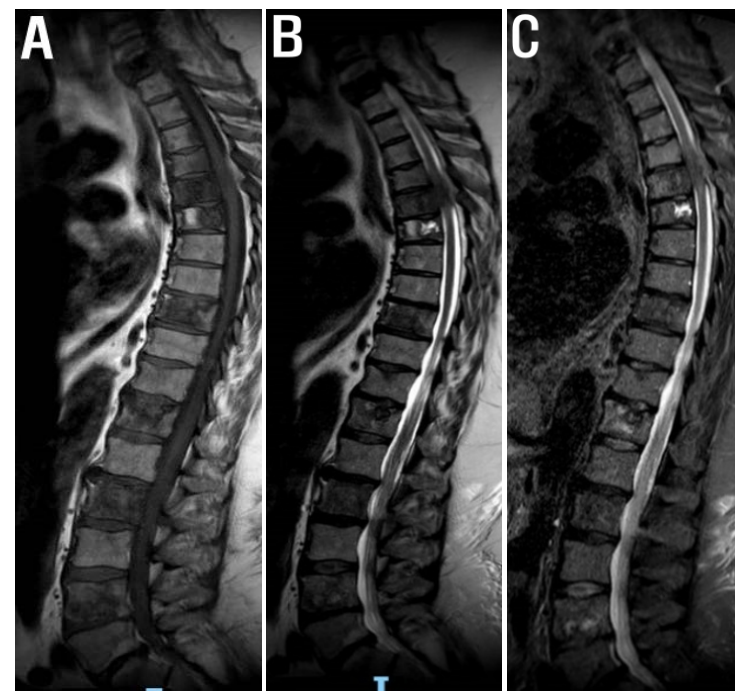

Figure 4. Sagittal full length spine T1 weighted (A), T2 weighted (B) and STIR (C) MR images shows multiple spinal metastasis originating from a prostate cancer.

neurological complications after deformity surgery. In multifocal spinal infections, entire spine should be visualized through MRI to evaluate the extension of infection and plan for the treatment. Axial SpA can be detected in early disease course with help of full spine MRI including SI joints. Metastasis to the spine is probably the most common indication for full length imaging as it allows evaluation by bone marrow change through whole spine MRI, especially in metastasis from prostate cancer and multiple myeloma. Due to the development of technology, taking time for CT and MRI of entire spine is being shorter and the resolution is increasing, and in particular, in the case of CT, the radiation exposure is gradually reducing. These changes will expand the indications for examining full length and help to diagnose, treat and follow up the patients with spine trauma and disease.

\section{Conflicts of Interest}

The authors declare no conflicts of interest regarding the publication of this paper.

\section{References}

[1] Døhn, U.M., Ejbjerg, B.J., Hasselquist, M., Narvestad, E., Møller, J., Thomsen, H.S. and Østergaard, M. (2008) Detection of Bone Erosions in Rheumatoid Arthritis Wrist Joints with Magnetic Resonance Imaging, Computed Tomography and Radiography. Arthritis Research \& Therapy, 10, R25. https://doi.org/10.1186/ar2378

[2] Imhof, H. and Fuchsjäger, M. (2002) Traumatic Injuries: Imaging of Spinal Injuries. European Radiology, 12, 1262-1272. https://doi.org/10.1007/s00330-002-1448-5

[3] Linsenmaier, U., Krötz, M., Häuser, H., Rock, C., Rieger, J., Bohndorf, K., Pfeifer, K. and Reiser, M. (2002) Whole-Body Computed Tomography in Polytrauma: Techniques and Management European Radiology, 12, 1728-1740.

https://doi.org/10.1007/s00330-001-1225-x 
[4] Balasubramanya, R. and Selvarajan, S.K. (2020) Lumbar Spine Imaging. StatPearls Publishing, Treasure Island.

[5] Anderson, S., Biros, M.H. and Reardon, R.F. (1996) Delayed Diagnosis of Thoracolumbar Fractures in Multiple-Trauma Patients Acad. Emergency Medicine, 3, 832839. https://doi.org/10.1111/j.1553-2712.1996.tb03527.x

[6] Takami, M., Nohda, K., Sakanaka, J., Nakamura, M. and Yoshida, M. (2014) Usefulness of Full Spine Computed Tomography in Cases of High-Energy Trauma: A Prospective Study. European Journal of Orthopaedic Surgery \& Traumatology, 24, 167-171. https://doi.org/10.1007/s00590-013-1268-0

[7] Deunk, J., Brink, M., Dekker, H.M., Kool, D.R., van Kuijk, C., Blickman, J.G., van Vugt, A.B. and Edwards, M.J. (2009) Routine versus Selective Computed Tomography of the Abdomen, Pelvis, and Lumbar Spine in Blunt Trauma: A Prospective Evaluation. Journal of Trauma and Acute Care Surgery, 66, 1108-1117.

https://doi.org/10.1097/TA.0b013e31817e55c3

[8] Atsina, K.-B., Rozenberg, A. and Selvarajan, S.K. (2019) The Utility of Whole Spine Survey MRI in Blunt Trauma Patients Sustaining Single Level or Contiguous Spinal Fractures. Emergency Radiology, 26, 493-500.

https://doi.org/10.1007/s10140-019-01693-0

[9] Kanna, R.M., Gaike, C.V., Mahesh, A., Shetty, A.P. and Rajasekaran, S. (2016) Multilevel Non-Contiguous Spinal Injuries: Incidence and Patterns Based on Whole Spine MRI. European Spine Journal, 25, 1163-1169. https://doi.org/10.1007/s00586-015-4209-2

[10] Youssef, J.A., Orndorff, D.O., Patty, C.A., Scott, M.A., Price, H.L., Hamlin, L.F., Williams, T.L., Uribe, J.S. and Deviren, V. (2013) Current Status of Adult Spinal Deformity Glob. The Spine Journal, 3, 51-62. https://doi.org/10.1055/s-0032-1326950

[11] Sucato, D.J. (2010) Management of Severe Spinal Deformity: Scoliosis and Kyphosis. Spine, 35, 2186-2192. https://doi.org/10.1097/BRS.0b013e3181feab19

[12] Kalra, M.K., Quick, P., Singh, S., Sandborg, M. and Persson, A. (2013) Whole Spine CT for Evaluation of Scoliosis in Children: Feasibility of Sub-Millisievert Scanning Protocol. Acta Radiologica, 54, 226-230. https://doi.org/10.1258/ar.2012.110625

[13] Lafon, Y., Lafage, V., Dubousset, J. and Skalli, W. (2009) Intraoperative ThreeDimensional Correction during Rod Rotation Technique. Spine, 34, 512-519. https://doi.org/10.1097/BRS.0b013e31819413ec

[14] Liau, K.M., Yusof, M.I., Abdullah, M.S., Abdullah, S. and Yusof, A.H. (2006) Computed Tomographic Morphometry of Thoracic Pedicles: Safety Margin of Transpedicular Screw Fixation in Malaysian Malay Population. Spine, 31, E545-E550. https://doi.org/10.1097/01.brs.0000225978.97652.e0

[15] Kim, H., Kim, H.S., Moon, E.S., Yoon, C.-S., Chung, T.-S., Song, H.-T., Suh, J.-S., Lee, Y.H. and Kim, S. (2010) Scoliosis Imaging: What Radiologists Should Know. RadioGraphics, 30, 1823-1842. https://doi.org/10.1148/rg.307105061

[16] Smith, J.A. (2002) Adult Deformity: Management of Sagittal Plane Deformity in Revision Adult Spine Surgery Contemp. Spine Surgery, 3, 10-16. https://doi.org/10.1097/01075922-200202000-00001

[17] Ozturk, C., Karadereler, S., Ornek, I., Enercan, M., Ganiyusufoglu, K. and Hamzaoglu, A. (2010) The Role of Routine Magnetic Resonance Imaging in the Preoperative Evaluation of Adolescent Idiopathic Scoliosis. International Orthopaedics, 34, 543-546. https://doi.org/10.1007/s00264-009-0817-y

[18] Kouijzer, I.J.E., Scheper, H., de Rooy, J.W.J., Bloem, J.L., Janssen, M.J.R., van den 
Hoven, L., Hosman, A.J.F., Visser, L.G., Oyen, W.J.G., Bleeker-Rovers, C.P. and de Geus-Oei, L.-F. (2018) The Diagnostic Value of 18F-FDG-PET/CT and MRI in Suspected Vertebral Osteomyelitis-A Prospective Study. European Journal of Nuclear Medicine and Molecular Imaging, 45, 798-805. https://doi.org/10.1007/s00259-017-3912-0

[19] Tins, B.J. and Cassar-Pullicino, V.N. (2004) MR Imaging of Spinal Infection Seminars. Musculoskeletal Radiology, 8, 215-229. https://doi.org/10.1055/s-2004-835362

[20] Cox, M., Curtis, B., Patel, M., Babatunde, V. and Flanders, A.E. (2018) Utility of Sagittal MR Imaging of the Whole Spine in Cases of Known or Suspected Single-Level Spinal Infection: Overkill or Good Clinical Practice? Clinical Imaging, 51, 98-103. https://doi.org/10.1016/j.clinimag.2018.02.009

[21] Moorthy, S. and Prabhu, N.K. (2002) Spectrum of MR Imaging Findings in Spinal Tuberculosis. American Journal of Roentgenology, 179, 979-983.

https://doi.org/10.2214/ajr.179.4.1790979

[22] Mann, S., Schütze, M., Sola, S. and Piek, J. (2004) Nonspecific Pyogenic Spondylodiscitis: Clinical Manifestations, Surgical Treatment, and Outcome in 24 Patients. Neurosurgical Focus, 17, 1-7. https://doi.org/10.3171/foc.2004.17.6.3

[23] Ledermann, H.P., Schweitzer, M.E., Morrison, W.B. and Carrino, J.A. (2003) MR Imaging Findings in Spinal Infections: Rules or Myths? Radiology, 228, 506-514. https://doi.org/10.1148/radiol.2282020752

[24] Kaila, R., Malhi, A.M., Mahmood, B. and Saifuddin, A. (2007) The Incidence of Multiple Level Noncontiguous Vertebral Tuberculosis Detected Using Whole Spine MRI. Clinical Spine Surgery, 20, 78-81. https://doi.org/10.1097/01.bsd.0000211250.82823.0f

[25] Siddiqui, M.A., Sartaj, S., Rizvi, S.W.A., Khan, M.J. and Khan, I.A. (2018) Role of Whole-Spine Screening Magnetic Resonance Imaging Using Short Tau Inversion Recovery or Fat-Suppressed T2 Fast Spin Echo Sequences for Detecting Noncontiguous Multiple-Level Spinal Tuberculosis. Asian Spine Journal, 12, 686. https://doi.org/10.31616/asj.2018.12.4.686

[26] Spernovasilis, N., Demetriou, S., Bachlitzanaki, M., Gialamas, I., Alpantaki, K., Hamilos, G., Karantanas, A. and Gikas, A. (2017) Characteristics and Predictors of Outcome of Spontaneous Spinal Epidural Abscesses Treated Conservatively: A Retrospective Cohort Study in a Referral Center. Clinical Neurology and Neurosurgery, 156, 11-17. https://doi.org/10.1016/j.clineuro.2017.02.019

[27] Braun, J. and Van Der Heijde, D. (2002) Imaging and Scoring in Ankylosing Spondylitis. Best Practice \& Research: Clinical Rheumatology, 16, 573-604. https://doi.org/10.1053/berh.2002.0250

[28] Barakat, E., Kirchgesner, T., Triqueneaux, P., Galant, C., Stoenoiu, M. and Lecouvet, F.E. (2018) Whole-Body Magnetic Resonance Imaging in Rheumatic and Systemic Diseases: From Emerging to Validated Indications. Magnetic Resonance Imaging Clinics, 26, 581-597. https://doi.org/10.1016/j.mric.2018.06.005

[29] Bennett, A.N., Marzo-Ortega, H., Rehman, A., Emery, P. and McGonagle, D. (2010) The Evidence for Whole-Spine MRI in the Assessment of Axial Spondyloarthropathy. Rheumatology, 49, 426-432. https://doi.org/10.1093/rheumatology/kep427

[30] Song, I.H., Hermann, K.G., Haibel, H., Althoff, C.E., Listing, J., Burmester, G.R., Krause, A., Bohl-Bühler, M., Freundlich, B. and Rudwaleit, M. (2011) Effects of Etanercept versus Sulfasalazine in Early Axial Spondyloarthritis on Active Inflammatory Lesions as Detected by Whole-Body MRI (ESTHER): A 48-Week Randomised Controlled Trial. Annals of the Rheumatic Diseases, 70, 590-596. 
https://doi.org/10.1136/ard.2010.139667

[31] Buhmann, S., Becker, C., Duerr, H.R., Reiser, M. and Baur-Melnyk, A. (2009) Detection of Osseous Metastases of the Spine: Comparison of High Resolution Multi-Detector-CT with MRI. European Journal of Radiology, 69, 567-573. https://doi.org/10.1016/j.ejrad.2007.11.039

[32] Shah, L.M. and Salzman, K.L. (2011) Imaging of Spinal Metastatic Disease. International Journal of Surgical Oncology, 2011, Article ID: 769753. https://doi.org/10.1155/2011/769753

[33] Lecouvet, F.E. (2016) Whole-Body MR Imaging: Musculoskeletal Applications. Radiology, 279, 345-365. https://doi.org/10.1148/radiol.2016142084

[34] Guillevin, R., Vallee, J.-N., Lafitte, F., Menuel, C., Duverneuil, N.-M. and Chiras, J. (2007) Spine Metastasis Imaging: Review of the Literature. Journal of Neuroradiology, 34, 311-321. https://doi.org/10.1016/j.neurad.2007.05.003 